Background We use published evidence to measure the uptake of national public health guidance recommendations. Available sources of uptake include national audits and reports, and peer reviewed journal articles. These are summarised and uploaded to an online database. This database is used to inform several streams of work in our organisation, including an internal review decision process for public health guidance. Identifying sources of uptake information in this area is challenging.

Objectives To assess the existing information on the database, and report on the effectiveness of a new approach to identifying potential sources of uptake information.

Methods An evaluation of public health uptake sources on the database was conducted, and a stakeholder mapping tool developed, which was used to systematically search for sources of uptake information. Stakeholders were contacted to provide information. Existing literature search strategies were reviewed and revised. Following these actions, the database was updated with the new sources and the impact of the exercise assessed.

Results The evaluation of the current database highlighted significant gaps regarding information relating to the uptake of public health guidance. Use of the methods outlined above identified a substantial amount of new information and a large number of potential sources of uptake for future reference.

Discussion The uptake of public health guidance is increasingly in the spotlight for the NHS, and knowing if recommendations have been implemented is helpful when deciding if guidance needs to be reviewed and updated. It is therefore important to have an accurate picture of uptake.

\section{P174 A SYSTEMATIC REVIEW OF TRANSCATHETER AORTIC VALVE IMPLANTATION}

Y Jung, M Choi. National Evidence-based Healthcare Collaborating Agency, Seoul, Korea

\section{0:1136/bmjqs-2013-002293.192}

Background Transcather Aortic Valve Implantation (TAVI) was developed as an alternative to surgical aortic valve replacement (AVR) for patients with severe symptomatic aortic stenosis (AS) and high or unacceptable surgical risk.

Objectives To evaluate the safety and effectiveness of TAVI compared with AVR or standard therapy.

Methods The searches were conducted via electronic databases including MEDLINE, EMBASE and the Cochrane Library and retrieved 1537 non-duplicate citations. Total 17 studies (2 RCT, 5 non-RCT, 10 cohort studies) were included for this review. Results Compared with standard therapy, TAVI significantly increased major stroke (risk ratio, 3.91; 95\% CI, 1.16-13.22) in two studies, although rate of major stroke was not significantly different in the TAVI compared with surgical AVR. Compared with standard therapy in inoperable patients, TAVI significantly reduced the all-cause mortality (risk ratio, 0.045, 95\% CI, 0.26-0.77) at 1 year and improved functional status (NYHA functional classification). Among high-risk patients, the mortality was not significantly different in the TAVI compared with surgical AVR. However, a RCT of 699 high-risk patients who were randomised to treatment either by TAVI or by surgical AVR reported that the all-cause mortality at 1 year was $24.2 \%$ and $26.8 \%$, respectively and TAVI was non-inferior to surgical $\operatorname{AVR}(\mathrm{p}=0.44)$.

Conclusion On the basis of current data, we recommend that TAVI is possible treatments as an alternative to surgical AVR for patients with AS who are considered to be inoperable or high risk for surgical AVR.

\section{P179 FACILITATING IMPLEMENTATION OF GUIDELINES FOR THE PREVENTION OF VASCULAR DISEASE IN GENERAL PRACTICE}

${ }^{1} \mathrm{M}$ Harris, ${ }^{2} \mathrm{~L}$ Litt, ${ }^{3} \mathrm{G}$ Russell, ${ }^{3} \mathrm{D}$ Mazza, ${ }^{1} \mathrm{~J}$ Lloyd, ${ }^{4} \mathrm{~N}$ Zwar, ${ }^{4}$ Taylor, ${ }^{5} \mathrm{M}$ Van Driel, ${ }^{6} \mathrm{C}$ Del Mar, ${ }^{7} Y$ Krastev, ${ }^{1} \mathrm{~S}$ Parker, ${ }^{6} \mathrm{~J}$ Smith. ${ }^{1}$ Centre for Primary Health Care and Equity, University of New South Wales, Sydney, Australia; '2Discipline of General Practice, Flinders University, Adelaide, Australia; ${ }^{3}$ School of Primary Health Care, Monash University, Melbourne, Australia; ${ }^{4}$ School of Public Health and Community Medicine, University of New South Wales, Sydney, Australia; ${ }^{5}$ Discipline of General Practice, University of Queensland, Brisbane, Australia; ${ }^{6}$ Faculty of Health Sciences and Medicine, Bond University, Gold Coast, Australia; 'Ethics Secretariate, University of Technology, Sydney, Australia

\section{0:1136/bmjas-2013-002293.193}

Background Although evidence based guidelines have been developed and disseminated, up to a half of patients do not receive guideline based preventive care.

Objectives This study aims to evaluate a model for the implementation of preventive care guidelines in general practice.

Methods Following a development process for the intervention involving a mixed method study and a pilot carried out in three practices a cluster randomised controlled trial is being conducted in 31 practices across four states. The intervention involves training, preventive care audit, and visits from a facilitator based in the local primary care support organisation. The facilitator assists practices to review their clinical audit and implement a practice plan structured around the 5As to improve the reach and quality of preventive care. Quantitative and qualitative evaluation methods are being used to assess impact on planned change within the practice, recalled and recorded preventive care, and patient behaviours and risk factors for cardiovascular disease.

Results Baseline data collection has been completed from practice staff and patients and the intervention is now complete. The recorded and patient recalled preventive care varied within and between practices resulting in a varied set of priorities for improvement. Early findings suggest that facilitation visits to review and plan improvements to the implementation of preventive guidelines are feasible, acceptable and can support organisational strategies to address gaps in care.

Discussion Our results may provide a model for local primary care support organisations to assist practices to improve their quality of preventive care.

\section{P180 SYSTEMATIC REVIEW OF CONTINUOUS INTERSCALENE BRACHIAL PLEXUS BLOCK FOR THE SHOULDER OR HUMERUS SURGERY PATIENTS}

${ }^{1} \mathrm{M}$ Choi, ${ }^{2} \mathrm{Y} \mathrm{Ko},{ }^{3} \mathrm{~J}$ Yeon, ${ }^{4} \mathrm{H}$ Oh, ${ }^{5} \mathrm{~S}$ Han. ${ }^{1}$ Department of Health Technology Assessment, National Evidence-based Collaboration, Seoul, Korea; ${ }^{2}$ Department of Rehabilitation of Medicine, Seoul Catholic Hospital, Seoul, Korea; ${ }^{3}$ Department of Rehabilitation of Anaesthesiology, Paik Hospital, Seoul, Korea; ${ }^{4}$ Department of Orthopedic Surgery, National Health Insurance Ilsan Hosapital, Seoul, Korea, ${ }^{5}$ Department of Orthopedic Surgery, Samsung Medical Center, Seoul, Korea

\section{0:1136/bmjqs-2013-002293.194}

Objective The safety and effectiveness of continuous interscalene brachial plexus block for the shoulder or humerus surgery. 
Methods Continuous interscalene brachial plexus block for the shoulder or humerus surgery patients was assessed using 8 domestic databases including Korea Med and Ovid-MEDLINE, Ovid-EMBASE and Cochrane Library. Through a search strategy, a total of 348 works were identified and total of 21 works were included in the final assessment. Two reviewers screened all references independently, for assessing included articles quality and extracted data.

Results Side effects of the drug were reported to have occurred less or at similar level in the intervention group than the control group, although procedure related complications were reported to have occurred more often in the intervention group compared to the control group. The average pain score following the surgery was reported to be significantly lower or similar for the intervention group compared with the control group. The intervention group also had lower or similar level of quantity of additional analgesics used in comparison to the control group. In addition, the intervention group displayed either similar or higher level of satisfaction of patients on the pain control method.

Conclusion Continuous interscalene brachial plexus block was assessed to be a safe and effective technology when used for the purpose of reduction in pain in shoulder or humerus surgery patients since it was found to be relatively safe when compared with the existing procedures, and similar or more effective in terms of pain control and usage of additional analgesics in comparison to the existing pain control methods.

\section{P181 SNORING, GASPING AND ADAPTE!}

D Bruinvels. Netherlands Society of Occupational Medicine - Centre of Excellence, Utrecht, The Netherlands

\section{0:1136/bmjqs-2013-002293.195}

Background The Netherlands Society of Occupational Medicine (NVAB) has a tradition of guideline development since 1999. The development of a typical monodisciplinary guideline for occupational physicians takes two years. In order to save time and costs the NVAB initiated an ADAPTE process for a monodisciplinary guideline on Obstructive Sleep Apnea Syndrome (OSAS).

Context OSAS is a major problem in the working population and may attribute to a substantial proportion of work related problems associated with fatigue. Examples are work accidents, work errors and productivity loss. Although a Dutch multidisciplinary guideline on OSAS exists, only a minority of the Dutch occupational physicians actually uses this guideline. To facilitate the implementation of the guideline, the NVAB provided a summary, a PowerPoint presentation, and medical case studies for occupational physicians. However, the guideline was still not implemented in clinical practice. Therefore the NVAB took the initiative to develop a monodisciplinary practice guideline for occupational physicians.

Description The ADAPTE process was used to develop a monodisciplinary practice guideline for occupational physicians based on a Dutch multidisciplinary guideline developed by $\mathrm{CBO}$ in 2009. The aim was to develop a monodisciplinary guideline in one year. In the end it took almost 3 years to adapt the guideline.

Lessons ADAPTE is a practical tool to adapt multidisciplinary guidelines into monodisciplinary practice guidelines. However, the use of the ADAPTE process does not guarantee time savings and reduction of development costs.

\section{P184 INCLUDING WORK PARTICIPATION IN DUTCH MULTIDISCIPLINAIRY GUIDELINES - AN OVERVIEW}

D Bruinvels. Netherlands Society of Occupational Medicine - Centre of Excellence, Utrecht, The Netherlands

\section{0:1136/bmjqs-2013-002293.196}

Background In 2006 an innovative programme for the development of multidisciplinary guidelines (KKCZ) was started in The Netherlands. The KKCZ programme aimed to develop multidisciplinary guidelines, to facilitate collaboration between professional groups, to enhance patient participation, and to avoid duplication of efforts on a national level. Context One of the innovative aspects of the KKCZ-programme was to stimulate guideline developers to include (work) participation in the guidelines. Therefore The Netherlands Society of Occupational Medicine (NVAB) has developed a "Blueprint Participation in Guidelines" to facilitate guideline developers to include work participation in KKCZ-guidelines.

Description The KKCZ-programme has led to 53 multidisciplinary guidelines. In an evaluation study the contents of eight KKCZ-guidelines and two other multidisciplinary guidelines was assessed. This was accomplished by first developing a list of process indicators describing essential steps of the Blueprint. Second, the use of the Blueprint was evaluated using this list. All eight KKCZ-guidelines were developed using the Blueprint. Because process indicators do not give information on the actual contents of a guideline, a web based survey was held among Dutch occupational physicians. 253 occupational physicians reported on the inclusion of work participation in the guidelines. The majority found that work participation was included in these guidelines. However, they also found that many of the key recommendations in the guidelines were difficult to implement.

Lessons The use of a Blueprint for guideline developers has stimulated the inclusion of work participation in multidisciplinary guidelines. However, the use of this Blueprint is no guarantee that key recommendations on work participation are implemented in daily practice.

\section{P187 DEVELOPING A FRAMEWORK AND CRITERIA TO SORT, SELECT AND REDUCE MEASUREMENT INSTRUMENTS FOR DAILY PRACTICE IN PHYSICAL THERAPY}

${ }^{1} G$ Meerhoff, , ${ }^{2} S$ Beurskens, ${ }^{2} R$ Swinkels, ${ }^{4} \mathrm{P}$ van der Wees, ${ }^{3} \mathrm{~V}$ de Graaf-Peters. ${ }^{1}$ Royal Dutch Society for Physical Therapy (KNGF), Amersfoort, The Netherlands; 'Zuyd University of Applied Sciences, Heerlen, The Netherlands; ${ }^{3}$ Hanze University of Applied Sciences, Groningen, The Netherlands; ${ }^{4}$ Scientific Institute for Quality of Healthcare (IQ), Nijmegen, The Netherlands

\section{0:1136/bmjqs-2013-002293.197}

Objectives The amount and diversity of measurement instruments in the Dutch physiotherapy guidelines is too extensive: in total 111 instruments. Many of these instruments are too long and concern identical domains and concepts. The aim of this bottom-up project is to develop a framework and criteria that will guide the structure, selection and reduction of instruments for application in daily practice.

Methods The project consist of the next steps: 1. Defining the aim of the framework and review the (inter)national literature; 2. Developing a concept framework; 3 . An inventory of domains and overlap of instruments in the Dutch guidelines; 4. An inventory made by professionals of domains and concepts relevant for musculoskeletal functioning in physiotherapy and instruments used; 5. Consensus meetings with representatives of 